\section{Complicações crônicas dos diabéticos tipo 2 atendidos nas Unidades de Saúde da Família, Recife, Pernambuco, Brasil}

\section{Chronic complications of type 2 diabetic attended at Family Health Units in the City of Recife, State of Pernambuco, Brazil}

Isabel Cristina Ramos Vieira Santos 1 Eduardo Freese de Carvalho 2 Wayner Vieira de Souza 3 Maria Carolina Wanderley Costa de Medeiros 4 Milka Gabrielle de Lira Nóbrega 5 Patrícia Michelly Santos Lima 6

1-3 Centro de Pesquisas Aggeu Magalhães. Fundação Oswaldo Cruz. Av. Professor Moraes Rego, s. n Cidade Universitária. Recife, PE, Brasil. CEP: 50.670-420.

E-mail: tutornad@yahoo.com.br

4-6 Faculdade de Enfermagem Nossa Senhora das Graças Universidade de Pernambuco. Recife, PE, Brasil.

\section{Abstract}

Objectives: to analyze the complications associated with type 2 diabetes in patients seen by Family Health Units.

Methods: a study was conducted of the Family Health Units of the six health districts of the city of Recife, State of Pernambuco, Brazil, with a sample of 1374 records of diabetic patients registered on the Family Health Program. The variables used were: the occurrence of macro-and microvascular complications, age, sex, years of schooling, alcohol consumption, smoking, first and last glucose recorded.

Results: there was a higher rate of complications in women (71.5\%) compared to men (29.5\%). Of the total, $58.9 \%$ had at least one complication. The macrovascular complications corresponded to $95.6 \%$. The peripheral vascular disease was responsible for 92.1\%, when considered as a single complication. There was an association between the age of 66 years or more and macrovascular complications, with a prevalence of $37.8 \%$. Microvascular complications were half as common among women as among men (3.6 $\times 6.4)$ and this represents a statistically significant difference $(p=0.04)$. Smoking was shown to be associated with microvascular complications $(p<0001)$.

Conclusions: the results point to a need to strengthen, alongside basic health services, measures recommended by the Health department, aiming to reduce risk factors and the impact of complications.

Key words Diabetes mellitus, Chronic complications, Family health units

\section{Resumo}

Objetivos: analisar as complicações associadas ao diabetes tipo 2 em pacientes atendidos pelas Unidades de Saúde da Família.

Métodos: estudo realizado nas Unidades de Saúde da Família dos seis distritos sanitários de Recife, Pernambuco, Brasil, com uma amostra de 1374 prontuários de diabéticos cadastrados no Programa de Saúde da Familia. As variáveis utilizadas foram: ocorrência de complicações macro e microvasculares, idade, sexo, anos de estudo, consumo de álcool, tabagismo, primeira e última glicemia registrada.

Resultados: observou-se uma freqüencia de complicações mais elevada em mulheres (71,5\%) quando comparada a dos homens (29,5\%). Do total, $58,9 \%$ apresentava pelo menos uma complicação. As complicações macrovasculares corresponderam a 95,6\%. A doença vascular periférica foi responsável por 92,1\%, quando considerada como complicação única. Verificou-se associação entre a faixa etária de 66 anos a mais e complicações macrovasculares, com uma prevalência de 37,8\%. As complicações microvasculares nas mulheres correspondem à metade daquelas encontradas para os homens $(3,6 x$ $6,4)$, sendo significante esta diferença $(p=0,04) . O$ tabagismo mostrou-se associado a complicações microvasculares $(p<0,001)$.

Conclusões: os resultados apontam para necessidade de se reforçar, junto aos serviços básicos de saúde, medidas preconizadas pelo Ministério da Saúde, visando reduzir os fatores de risco e o impacto de suas complicações.

Palavras-chave Diabetes mellitus, Complicações crônicas, Unidades de saúde da família 


\section{Introdução}

O diabetes mellitus (DM) atinge em todo o mundo grande número de pessoas de diferentes condições sociais. ${ }^{1}$ É uma doença crônica grave, que integra um grupo de doenças metabólicas, caracterizada por níveis elevados de glicose no sangue (hiperglicemia) resultante de defeitos na secreção de insulina e/ou na ação desta.2,3

Essa enfermidade representa um problema de saúde pública e pessoal com grandes proporções quanto à magnitude e à transcendência, apesar dos progressos no campo da investigação e da atenção aos pacientes. 4,5

O diabetes está associado ao aumento da mortalidade devido ao alto risco de desenvolvimento de complicações agudas e crônicas. Nas primeiras estão listadas a hipoglicemia, cetoacidose diabética e o coma hiperosmolar. Já as complicações crônicas podem ser decorrentes de alterações na microcirculação, causando retinopatia e nefropatia; na macrocirculação, levando à cardiopatia isquêmica, doença cerebrovascular e doença vascular periférica e, ainda, neuropáticas. 5,6

O impacto do diabetes mellitus como problema de saúde pública deve-se principalmente às complicações crônicas decorrentes da doença que frequentemente tem caráter incapacitante, comprometendo a qualidade de vida dos seus portadores e requerendo tratamento extremamente oneroso ao sistema de saúde. 7,8

Nos Estados Unidos, metade de todas as pessoas com diabetes acima de 65 anos de idade são hospitalizadas a cada ano, e as complicações graves e com risco de vida contribuem para as taxas aumentadas de hospitalização. Os custos relacionados ao diabetes são estimados em cerca de US\$99 bilhões por ano, incluindo os gastos diretos com atenção médica e os custos indiretos atribuídos à incapacidade e a mortes prematuras. ${ }^{9,10}$

O casos de diabetes mellitus tipo 2 são frequentemente oligosintomáticos ou mesmo assintomáticos, podendo um paciente evoluir sem sintomas por vários anos e apresentar apenas ligeira hiperglicemia de jejum ou pós-prandial.2,11 Consequentemente, o diabetes frequentemente se manifesta por sinais e sintomas das complicações metabólicas agudas ou degenerativas e infecciosas secundários geradas pela doença. Os sintomas característicos da fase aguda são: poliúria, polidpsia, polifagia, emagrecimento, perda da força; e as principais complicações degenerativas são: infarto do miocárdio, arteriopatia periférica, acidente vascular cerebral (AVC), microangiopatia, nefropatia e neuropatia. 11,12
A neuropatia diabética raramente é determinante da causa mortis em diabéticos, porém ela é a mais prevalente das complicações crônicas em um serviço geral de assistência a diabéticos (cerca de $40 \%$ dos pacientes têm algum tipo de neuropatia), contribuindo em grande parte para a incapacitação que ocorre nas fases avançadas da doença. ${ }^{11,13}$

Entre as ações de enfrentamento ao diabetes atualmente desenvolvidas no Brasil, destacam-se as desenvolvidas pelo Programa Saúde da Família (PSF). Essas atividades vão desde o atendimento ambulatorial com a realização de consultas e outros procedimentos, até as visitas domiciliares, as ações de educação em saúde e vigilância epidemiológica, entre outras. ${ }^{8}$

O PSF contribui ainda como excelente fonte de dados sobre a população assistida, viabilizando assim a condução de estudos epidemiológicos que permitam conhecer a real dimensão do problema, proporcionando um acervo de dados úteis ao manejo da situação.

Considerando que indivíduos com diabetes mellitus podem permanecer sem diagnóstico durante longo tempo, fato que os tornam mais vulneráveis às alterações metabólicas e suas complicações crônicas e que cabe às unidades de saúde do Programa de Saúde da Família o papel de detectar precocemente essas complicações, o presente artigo tem por objetivos: investigar a freqüência e distribuição de complicações crônicas do diabetes tipo 2 na população e verificar a sua associação com características inerentes à pessoa, escolaridade e perfil glicêmico, em pacientes atendidos nas Unidades de Saúde da Família da cidade do Recife.

\section{Métodos}

Trata-se de um estudo epidemiológico, descritivo de corte transversal. A cidade do Recife, Pernambuco, com uma população estimada em 2004 de 1.486 .869 habitantes, com 1.422.905 pessoas residentes na área urbana, encontra-se dividida, do ponto de vista da assistência sanitária, em seis distritos. 14

Tendo em vista a área de abrangência do PSF no município, a instituição do diagnóstico precoce da diabetes mellitus na comunidade atendida, a cidade do Recife apresentou-se para esse estudo como local ideal à realização do levantamento.

Os dados relativos aos pacientes portadores de diabetes cadastrados no PSF foram obtidos a partir do Sistema de Informação da Atenção Básica (SIAB). Segundo esse sistema existia, em 2005, 13.396 pacientes cadastrados, com uma prevalência 
média de $3,7 \%$ nos seis distritos. A partir desses dados, foi realizado o cálculo amostral, estabelecendo-se o quantitativo de 1374 prontuários de pacientes diabéticos como suficiente para produzir as estimativas desejadas. Do total da amostra, 1055 pacientes tinham relato em prontuário quanto à ocorrência de complicações. Nessa amostra, 2/3 foi composta por mulheres a partir de 25 anos de idade.

Considerou-se como população alvo para o cálculo amostral os portadores de diabetes mellitus tipo 2 atendidos no ano de 2005, sendo alocados de forma proporcional ao número de diabéticos cadastrados em cada Distrito Sanitário.

O instrumento para coleta de dados foi um formulário elaborado pelos pesquisadores para a obtenção das informações dos prontuários dos pacientes nas respectivas Unidades de Saúde da Família (USF). O formulário constou de 16 questões relacionadas às variáveis de interesse, tais como idade, sexo, anos de estudo, consumo de álcool, tabagismo, primeira e última glicemia registrada. A coleta foi feita no período de março a julho de 2006.

De posse dos dados, a população foi classificada quanto à ocorrência ou não de complicações e em seguida, quanto à natureza das complicações: macro e microvasculares. Em seguida, a distribuição de freqüência da presença ou não de complicações e o tipo de complicações foi descrita segundo as variáveis de interesse. Testou-se a significância das associações através do teste do qui-quadrado, com um nível de significância de 5\%.

Os dados da pesquisa foram digitados em dupla entrada e processados em microcomputador, utilizando-se para tabulação e realização de testes estatísticos o software Statistical Package for the Social Sciences (SPSS), versão 11,0.

Todos os procedimentos relacionados à coleta $\mathrm{e}$ análise dos dados só foram iniciados após julgamento e aprovação do projeto pelo Comitê de Ética da Universidade de Pernambuco devidamente indicado pelo Sistema Nacional sobre Ética em Pesquisa (SISNEP).

\section{Resultados}

A prevalência de complicações crônicas encontrada nos pacientes atendidos nas Unidades de Saúde da Família pode ser observada na Figura 1. Verifica-se que $58,9 \%$ dos portadores de diabetes tipo 2 apresentava pelo menos uma complicação registrada em prontuário e 17,9\% duas ou mais complicações.

As complicações crônicas, segundo o sítio circulatório acometido, apresentaram uma prevalência de
Figura 1

Freqüência de complicações associadas ao quadro de diabetes tipo 2 em pacientes atendidos nas Unidades de Saúde da Família. Recife, 2007.

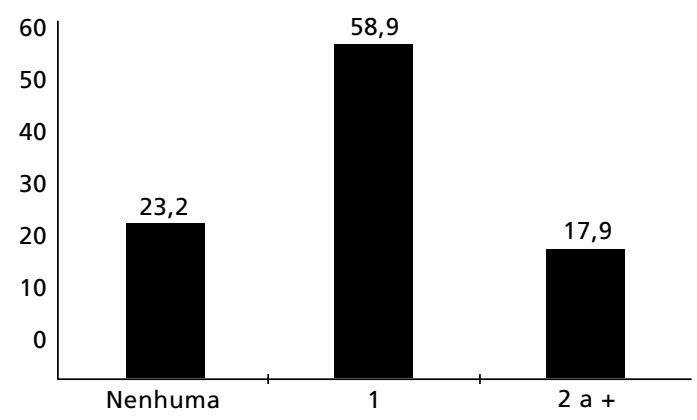

Tabela 1

Tipo de complicações observadas em portadores de diabetes mellitus 2 atendidos nas Unidades de Saúde da Família. Recife, 2007.

\begin{tabular}{lrr}
\hline Tipo de complicações crônicas & N & \% \\
\hline Macrovasculares & & \\
Doença vascular periférica & 929 & 92,1 \\
Cardiopatia e doença cerebrovascular & 32 & 3,2 \\
Doença cerebrovascular e neuropatia & 48 & 4,7 \\
Total & 1009 & 95,6 \\
Microvasculares & & \\
Retinopatia & 24 & 52,2 \\
Nefropatia & 15 & 32,6 \\
Retinopatia e Nefropatia & 7 & 15,2 \\
Total & 46 & 4,4 \\
Total & 1055 & 100,0 \\
\hline
\end{tabular}

complicações macrovasculares correspondentes a $95,6 \%$, entre as quais ressalta-se a ocorrência da doença vascular periférica, responsável por $92,1 \%$ dos casos desse grupo. As complicações microvasculares tiveram uma prevalência de $4,4 \%$, destacando-se as retinopatias com uma prevalência de $52,2 \%$ (Tabela 1 ).

A Tabela 2 mostra a ocorrência de complicações de acordo com as variáveis inerentes às pessoas (idade e sexo), anos de estudo, hábitos (consumo de álcool e tabagismo) e perfil glicêmico. A faixa etária mais prevalente foi a de 66 ou mais anos, sendo a diferença de idade significativa estatisticamente para as complicações macrovasculares $(p<0,05)$. Quanto ao sexo, observou-se uma maior prevalência dos 
Tabela 2

Características associadas ao tipo de complicações em portadores de diabetes mellitus 2 atendidos nas Unidades de Saúde da Família. Recife, 2007.

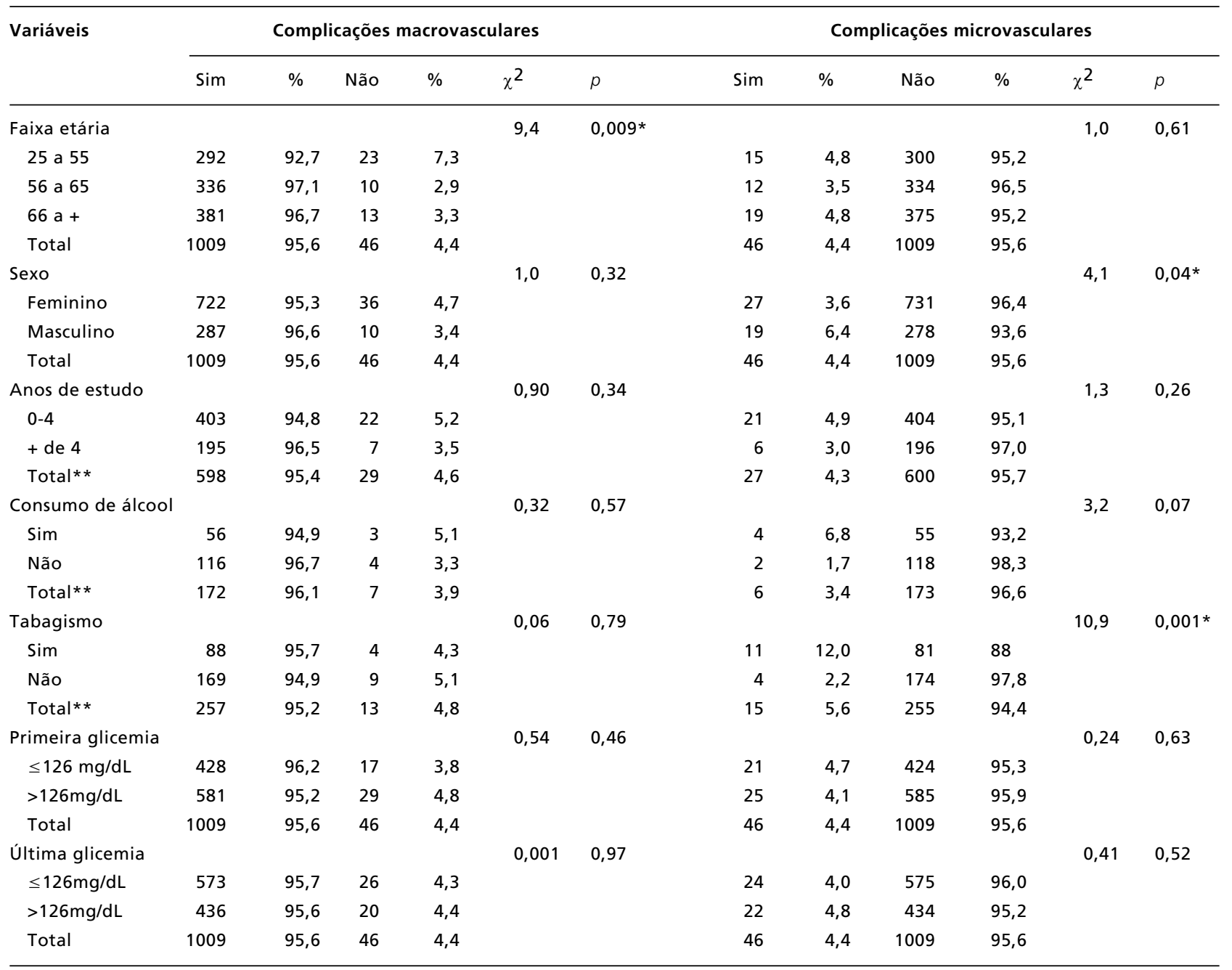

* $p<0,05 ; * *$ Número da amostra diminuído devido à falta de registro desses dados.

dois grupos de complicações (micro e macrocirculatória) no sexo feminino, encontrando-se significância estatística para o grupo de complicações microvasculares $(p<0,05)$. Quanto a este tipo de complicação, nota-se que sua ocorrência nas mulheres corresponde à metade da que se verifica para os homens $(3,6$ versus 6,4$)$ sendo significante essa diferença $(p=0,04)$.

Os resultados referentes à escolaridade, consumo de álcool e tabagismo estão reduzidos, devido à ausência de registro desses dados nos prontuários.

No que se refere à escolaridade, aqui categorizada por número de anos de estudo, a maior prevalência encontrada correspondeu à faixa de zero a quatro anos, e não se verificou significância estatística entre essa variável e a ocorrência de complicações crônicas. Quanto ao consumo de álcool, foi observada uma prevalência de $32,5 \%$ entre o grupo dos portadores de complicações macrovasculares e de $66,6 \%$ para os portadores de complicações microvasculares, não se verificando associação estatisticamente significativa.

Em relação ao tabagismo, observou-se uma prevalência de $34,3 \%$ no grupo de portadores de complicações macrovasculares e de $73,3 \%$ no de complicações microvasculares, verificando-se associação significativa $(p=0,001)$.

O perfil glicêmico mostra, na primeira glicemia 
registrada, uma maior prevalência de valores acima de $126 \mathrm{mg} / \mathrm{dL}$ para ambos os grupos, havendo modificação desse status no registro da última glicemia, também para os dois tipos de complicações. Para tais diferenças não foi observada significância estatística (Tabela 2).

\section{Discussão}

Observou-se neste estudo que mais da metade da amostra apresentou pelo menos uma complicação registrada em prontuário. Dessas, as complicações macrovasculares corresponderam a $95,6 \%$, nas quais a doença vascular periférica se destaca, responsável por $92,1 \%$ das complicações desse grupo.

Alguns estudos relatam que os pacientes com DM do tipo 2 têm um risco quatro vezes maior de ter doença vascular periférica (DVP) em relação a não diabéticos. 15,16 Segundo Oliveira, 17 pessoas com diabetes apresentam elevado risco de doença vascular aterosclerótica, como as doenças coronarianas, arterial periférica e vascular cerebral. As complicações crônicas do DM são as principais responsáveis pela morbidade e mortalidade dos pacientes diabéticos. As doenças cardiovasculares representam a principal causa de morte $(52 \%)$ em pacientes diabéticos do tipo 2.18 Essas complicações macroangiopáticas podem ocorrer mesmo em estágios precoces da doença e se apresentam de forma mais difusa e grave do que em pessoas sem essa condição. 18

De acordo com dados do Ministério da Saúde, 19 a DVP está presente em $8 \%$ dos diabéticos tipo 2, por ocasião do diagnóstico, e pode ocorrer em $45 \%$, após 20 anos da doença.

Observou-se neste estudo uma maior prevalência de complicações entre aqueles com 66 ou mais anos de idade em relação às complicações macrovasculares $(p<0,05)$. Esse achado é concordante com outros estudos realizados nas regiões Sul e Sudeste, ${ }^{20,21}$ que confirmam a tendência do aumento da prevalência de diabetes com o envelhecimento. $\mathrm{O}$ mesmo não se verificou para as complicações microvasculares.

A despeito do diabetes ser uma doença comum na população idosa, Barreto 22 afirma que cerca da metade dos casos não tem diagnóstico estabelecido, o que deixa o idoso exposto inicialmente à hiperinsulinemia e posteriormente à hiperglicemia e, em ambas as fases, às complicações crônicas delas decorrentes.

Ao contrário do resultado de estudo de prevalência realizado por Goldenberg et al. 23 em São
Paulo, no presente estudo se verificou associação entre o sexo feminino e a ocorrência de complicações microvasculares $(p<0,05)$, resultado consistente com aqueles alcançados por Freitas e Jorge20 em relação à mortalidade e morbidade hospitalar por diabetes mellitus na população idosa em um município da Região Sul do Brasil.

Franco et al., ${ }^{24}$ analisando os óbitos de pessoas com 40 anos e mais de idade, residentes no Estado de São Paulo, observaram que o DM representou aproximadamente $50 \%$ dos óbitos masculinos e cerca de $80 \%$ dos óbitos femininos no grupo das doenças endócrinas, nutricionais e metabólicas.

De fato, a literatura tem apontado para a variação na prevalência da doença entre os sexos, no tempo e no espaço. Nesse trabalho, a participação feminina, no que diz respeito à ocorrência de complicações crônicas do DM, foi expressiva na composição da amostra, quando observamos uma freqüência mais de duas vezes superior a dos homens. O fato de se ter encontrado associação positiva para o sexo feminino em relação às complicações microvasculares a princípio pode estar relacionado à maior preocupação das pessoas desse sexo em relação ao estado de saúde. Outro fato a ser considerado é que no Brasil, como em outras sociedades contemporâneas, a expectativa de vida é sempre mais elevada para as mulheres do que para os homens. No país, essa é de 67,6 anos para homens e de 75,2 para mulheres, em 2003.25 Consequentemente, aumentam as chances das mulheres apresentarem um percentual maior de complicações que os homens.

No que diz respeito à escolaridade e hábitos de vida, chama atenção o baixo índice de registro desses dados nos prontuários dos pacientes diabéticos atendidos no município. Tal fato limita o poder da análise em relação a essas variáveis e indica a possibilidade de outras inadequações na atenção prestada de prevenção e controle das complicações dos portadores de diabetes tipo 2 .

Não foi observada relação entre o número de anos de estudo e os dois grupos de complicações. Em estudo sobre diabetes auto-referido, Goldenberg et al. 23 encontraram uma prevalência de $39,8 \%$ com escolaridade acima de oito anos de estudo e referência positiva para doença, enquanto esta proporção foi de 22,6\% nos níveis abaixo dessa faixa.

Não se verificou associação entre consumo de álcool e as complicações diabéticas. Freitas, ${ }^{26} \mathrm{em}$ estudo recente sobre perfil bio-psicossocial da população diabética em uma comunidade do Ceará, trabalhando com uma amostra de 78 pacientes diabéticos, encontrou uma prevalência de $19,7 \%$ de consumo de álcool. De acordo com a literatura, esse 
hábito relaciona-se mais às complicações agudas como hipoglicemia e acidose metabólica. 27

Foi observada a associação entre tabagismo e complicações microvasculares $(p<0,001)$, em consonância com o estudo de Freitas, ${ }^{26}$ que encontrou uma prevalência de tabagismo de $54,1 \%$ dentro de uma amostra de 78 pacientes. Aqui a prevalência de complicações microvasculares relacionadas ao tabagismo foi de $73,3 \%$. Esse resultado supera aqueles encontrados por Scheffel et al., ${ }^{16}$ que em uma amostra de 927 pacientes com DM do tipo 2 encontrou uma prevalência de vinte e dois por cento de fumantes atuais e $21 \%$ de ex-tabagistas.

Quanto ao perfil glicêmico da população atendida pelo PSF na cidade do Recife, aqui categorizada pela primeira e última glicemia plasmática registradas em prontuário, ao contrário da literatura, não se verificou associação com a ocorrência de complicações. Segundo Bem e Kunde, 28 a dosagem da glicose no sangue não constitui parâmetro eficiente para avaliação do controle da glicemia durante um intervalo de tempo prolongado. Nesse sentido, a dosagem da hemoglobina glicada (HbA1c) tem papel fundamental na monitorização do controle glicêmico em pacientes diabéticos, pois fornece informações acerca do índice retrospectivo da glicose plasmática. 29

Embora o Ministério da Saúde6 estabeleça a hemoglobina glicada ou glicohemoglobina como parâmetro para o controle metabólico a longo prazo, essa prática não está ainda incorporada na atenção oferecida pelas unidades de saúde da cidade do Recife.

Considerando que: o DM é mais freqüentemente diagnosticado entre 45 e 55 anos de idade; que a população brasileira apresenta uma explícita tendência de ampliação na expectativa de vida, e que vários estudos tem mostrado demonstram que a prevenção das complicações do DM é possível, os resultados desse estudo acenam para necessidade de se reforçar, junto aos serviços básicos de saúde, as medidas e ações, no âmbito municipal, preconizadas pelo Ministério da Saúde, visando reduzir os fatores de risco e o impacto dessas complicações na qualidade de vida dos portadores de diabetes Tipo 2, para que os mesmos tenham uma forma mais saudável e participativa na sociedade.

\section{Agradecimentos}

Ao Fundo de Desenvolvimento de Pesquisa da Universidade de Pernambuco (FDPE), pela concessão de bolsa de iniciação científica. À Secretaria de Saúde da Prefeitura da cidade do Recife pela viabilização da coleta de dados. A Roseane Almeida da Silva pela colaboração na correção das referências bibliográficas.

\section{Referências}

1. Assunção MCF, Santos IS, Gigante DP. Atenção primária em diabetes no Sul do Brasil: estrutura, processo e resultado. Rev Saúde Pública [serial online] 2001; 35: 88-95. [acesso em: 20 mar. 2005]. Disponível em: <http://www. scielo.br/scielo.php?script=sci_arttext\&pid=S0034-891020 $01000100013 \& \operatorname{lng}=\mathrm{en} \& \mathrm{nrm}=\mathrm{iso}>$.

2. Fontbonne A, Freese E. Epidemiologia do diabetes tipo 2 e a resistência à insulina. In: Freese E. Epidemiologia, políticas e determinantes das doenças crônicas não transmissíveis no Brasil. Recife: UFPE; 2006. p. 159-176.

3. Horton R. The neglected epidemic of chronic disease. Lancet. 2005; 366: 1514-24.

4. Christopher JL, Murray ADL. Mortality by cause for eight regions of the world: Global Burden of Disease Study. Lancet. 1997; 349: 1040-54.

5. Strong K, Mathers C, Leeder S, Beaglehole R. Preventing chronic diseases: how many lives can we save? Lancet. 2005; 366: 1578-82.

6. Brasil. Ministério da Saúde. Secretaria de Políticas de Saúde. Departamento de Ações Programáticas Estratégicas. Plano de reorganização da atenção à hipertensão arterial e ao diabetes mellitus. Brasilia, DF; 2002.
7. Selli L, Papaléo LK, Meneghel SN, Tornero JZ. Técnicas educacionales en el tratamiento de la diabetes. Cad Saúde Pública. 2005; 21: 1366-72.

8. Brasil. Ministério da Saúde. Manual de enfermagem: Programa Saúde da Família. São Paulo; 2001.

9. Barros MBA, César CLG, Carandina L, Torre GD. Desigualdades sociais na prevalência de doenças crônicas no Brasil, PNAD-2003. Ciência Saúde Colet. 2006; 11: 911-26.

10. Smeltzer SC, Bare BG. Histórico e tratamento de pacientes com diabetes mellitus. In: Smeltzer SC, Bare BG. Brunner/Suddarth: tratado de enfermagem médico cirúrgica.. 9. ed. Rio de Janeiro: Guanabara Koogan; 2002. Cap. 37, p. 933-83.

11. Brasil. Ministério da Saúde. Manual do diabetes. 2. ed. Brasília, DF; 1993.

12. Costa JSD, Olinto MTA, Assunção MCF, Gigante DP, Macedo S, Menezes AMB. Prevalência de diabetes mellitus em Pelotas, RS: um estudo de base populacional. Rev Saúde Pública. 2006; 40: 542-5.

13. Lopes CF. Pé diabético. In: Pitta GBB, Castro AA, Burihan E, editores. Angiologia e cirurgia vascular: guia ilustrado. Maceió: UNCISA; 2003. 
14. IBGE (Instituto Brasileiro de Geografia e Estatística). Departamento de População e Indicadores Sociais. Síntese de indicadores sociais 2004. Rio de Janeiro; 2005.

15. CDC (Center for Disease Control and Prevention). Diabetes-related amputations of lower extremities in the medicare population-Minnesota, 1993-1995. MMWR Morb Mortal Wkly Rep. 1998; 47: 649-52.

16. Scheffel RS, Bortolanza D, Weber CS, Da Costa LA, Canani LH, Santos KG, Crispim D, Roisenberg I, Lisbôa HRK, Três GS, Tschiedel B, Gross JL. Prevalência de complicações micro e macrovasculares e de seus fatores de risco em pacientes com diabetes melito do tipo 2 em atendimento ambulatorial. Rev Assoc Med Bras. 2004; 50: 263-7.

17. Oliveira JEP. Diagnóstico e classificação do diabetes melito e tratamento do diabetes melito do tipo 2. In: Sociedade Brasileira de Diabetes. Consenso brasileiro sobre diabetes 2002. Rio de Janeiro: Diagraphic; 2003. p. 72-94.

18. Nathan DM, Meigs J, Singer DE. The epidemiology of cardiovascular disease in type 2 diabetes mellitus: how sweet is it? Lancet. 1997; 350 (Suppl.1): 14-9.

19. Brasil. Ministério da Saúde. Secretaria de Políticas de Saúde. Plano de reorganização da atenção à hipertensão arterial e ao diabetes mellitus. Brasília, DF; 2001.

20. Freitas MTA, Jorge MHPM.. Diabetes mellitus na população idosa em município da Região Sul do Brasil: um estudo da mortalidade e morbidade hospitalar. Arq Bras Endocrinol Metab. [serial online]. 2004; 48: 505-12. [acesso em: 20 abr. 2007]. Disponível em: http://www.scielo.br/scielo.php?script=sciarttex\&pid=S000 4-2730200400011\&lng= pt\&nrm=iso

21. Passos VMA, Barreto SM, Diniz LM, Lima-Costa MF. Diabetes tipo 2: prevalência e fatores associados em uma comunidade brasileira. Projeto Bambuí de estudo de saúde e envelhecimento. São Paulo Med J. [serial online] 2005; 123: 66-71. [acesso em 20 abr. 2007]. Disponível em: http://www.scielo.br/scielo.php?script=sciarttext\&pid=S15 16-31802005000200007\&lng=pt\& nrm $=$ iso

Recebido em 17 de agosto de 2007

Versão final apresentada em 3 de março de 2008

Aprovado em 5 de março de 2008
22. Barreto NDM. Diabetes mellitus na pessoa idosa. Arq Geriatr Gerontol. 1997; 1: 65-71.

23. Goldenberg P, Schenkman S, Franco LJ. Prevalência de diabetes mellitus: diferenças de gênero e igualdade entre os sexos. Rev Bras Epidemiol. 2003; 6: 18-28.

24. Franco LJ, Mameri C, Pagliaro H, Iochida LC, Goldenberg P. Diabetes como causa básica ou associada de morte no Estado de São Paulo, Brasil, 1992. Rev Saúde Pública. 1998; 32: 237-45.

25. IBGE (Instituto Brasileiro de Geografia e Estatística). Departamento de População e Indicadores Sociais. Tábuas completas de mortalidade: 2003. [monografia online]. [acesso em: 8 nov. 2007]. Disponível em: $<$ http://www.ibge. mgov.br/home/presidencia/noticias /noticia_visualiza.php?id_noticia=266\&id_pagina $=1>$

26. Freitas IMP. Estudo do perfil bio-psicossocial da população diabética na comunidade do dendê cadastrada no NAMI [dissertação]. Fortaleza: Centro de Ciências da Saúde da Universidade de Fortaleza; 2005.

27. Santana MG, organizador. Rede de saberes em diabetes e saúde: um exercício de interdisciplinaridade. Pelotas: Independente; 2002.

28. Bem AF, Kunde J. A importância da determinação da hemoglobina glicada no monitoramento das complicações crônicas do diabetes mellitus. J Bras Patol Med Lab. 2006; 42: $185-91$.

29. Sacks DB, Bruns DE, Goldstein DE, Maclaren NK, McDonald JM, Parrott M. Guidelines and recommendations for laboratory analysis in the diagnosis and management of diabetes mellitus. Clin Chem. 2002; 48: 436-72. 